\title{
ARTICLE
}

\section{Result of study on developing forest seed region in Mongolia}

\author{
Jamyansuren S. ${ }^{1}$ Udval B. ${ }^{2 *}$, Batkhuu ${ }^{3}{ }^{3}$, Bat-Erdene.$^{4}{ }^{4}$ and Michael Fischer ${ }^{5}$ \\ ${ }^{1}$ Institute General and Experimental Biology, Mongolian Academy of Sciences, \\ Ulaanbaatar, Mongolia \\ ${ }^{2}$ Institute of Geography and Geoecology, Mongolian Academy of Sciences, \\ Ulaanbaatar, Mongolia \\ ${ }^{3}$ School of Engineering and Applied Science, National University of Mongolia, \\ Ulaanbaatar, Mongolia \\ ${ }^{4}$ School of Agroecology, Mongolian University of Life Sciences, \\ Ulaanbaatar, Mongolia \\ ${ }^{5}$ Asian Development Bank, Manila, Philippines
}

\begin{abstract}
In foreign countries, motor roads and railroads are usually used as borders of forest seed regions. In the case of Mongolia, this method is not suitable in view of the sparse population, large distribution area of forests and the huge territory of the country. Therefore, this study used topographical features to determine the borders of the seed regions. Satellite images in combination with Digital Elevation Model (DEM) make it possible to define rivers, streams and mountain ridges as the borders of forest seed regions. Overall, we identified 19 seed regions for Siberian larch and 12 regions for Scots pine, 9 regions for Siberian pine, 6 regions for Siberian fir and 9 seed regions for Siberian spruce forests. Due to a lack of genetic-selection studies in Mongolia, these proposed forest seed regions can be considered as a preliminary effort with an opportunity to be updated and improved based on more detailed research results. The forest seed regions and maps will play important role as fundamental material for establishing a permanent forest seed supply based on genetic-selection characteristics of the forests in different regions in Mongolia.
\end{abstract}

Keywords: Seed; Region; Pine; Larch; Siberian pine; Siberian fir; Siberian spruce;

\section{INTRODUCTION}

The forestry sector in Mongolia is rapidly changing, including reforestation and restoration

activities which been broadening in all aspects. Therefore, it is vitally important to establish scientific knowledge and understanding of the hereditary and genetic characteristics of the main forest-forming tree species for certain forest regions to ensure successful reforestation and forest restoration efforts. Hence, it is vital to determine forest seed regions in order to utilize geographic difference in adaptation and productivity of trees in order to grow high productivity forests which can withstand adverse environmental factors. 
Several previous researches, including Milyutin et al., (1988) produced preliminary forest seed zoning under the framework of Mongolian-Russian Joint Biological Complex Expeditions conducted in 19811990 and they identified 13 seed regions for Siberian larch and 4 seed regions for Scotch pine forests respectively [13]. These studies were notable in terms of their significance in improving the quality of reforestation work, enabling experimental research to use seeds from geographically different regions, and establishing permanent seed stands based on the genetic-selection process in each district [17]. In addition, the study into internal modification of larch species in eastern Khentii along with research to identify parameters of larch seed quality have helped determined the best regions (forest seed zoning) to transplant seeds across. The joint expeditions also

\section{MATERIALS AND METHODS}

The basic units of the seed region are the identical genetic origin and growth conditions, which differ from neighboring regions which is representative of a given provenance region in terms of altitude, climate, soil and vegetation characteristics. Local seeds are those that have been collected from forests within the border of the same seed region; and seeds from other regions refer to those that have been collected from other seed regions. The following materials, information and sources are used in developing seed regions and their mapping.

1. Digital elevation model of SRTM satellite data with $30 \mathrm{~m}$ accuracy

2. Satellite maps Google Earth

3. Meteorological data of study areas

4. Forest inventory reports, materials, and maps

5. Specialized maps
I. Topographic map
II. Digital elevation model
III. Mountain slope map
IV. Soil map

reported that the difference and modification in the morphological characteristics of larch populations were dependent on the growth conditions, and distribution of populations. What was new about this study was that it was able to determine the larch seed districts in the forest-vegetation zone of Khentii, where Larix sibirica, L. daurica, and $L$. chekanowskii are present $[1,5,6,7,9,10,11,12]$. The main objective of this study is to develop the seed region mapping of Mongolia's coniferous forests based on genetic-selection characteristics. For this purpose, we aim to identify seed regions for coniferous trees including Siberian larch (Larix sibirica Ldb.), Scots pine (Pinus sylvestris L.); Siberian pine (Pinus sibirica Du Tour.), Siberian fir (Abies sibirica Ldb.), and Siberian spruce (Picea obovata Ldb.)

\section{Vegetation map \\ VI. Forest distribution map}

The forest distribution map (shape file) with descriptions of 464,323 stands was developed by the Forest Research Development Center (FRDC) of the Ministry of Environment and Tourism (MET). These shape files had information on species composition, tree species, and ecological and geographical data of the forest stand and compartments. The digital elevation model, different satellite images, specialized thematic maps and field survey results on soil and vegetation characteristics have been used as additional materials.

A digital elevation model was used with Surpace to define aspects. Spatial overlapping analysis has been conducted to prepare and extract information from specialized maps [3,4,13,14].

Based on the experiences from other countries, motor roads and railroads are usually used as borders of the forest seed regions 
[16]. In the case of Mongolia, this method is not suitable due to the sparse population, large distribution area of forests and the large territory. Therefore, we used topographical features to determine borders of the seed regions. Topographical maps visibly denote the borders using river, stream and mountain ridges as border areas $[2,8,15,16]$.

\section{RESULTS AND DISCUSSION}

The basic unit of the seed regions is the forest seed region, which is characterized by similar soil and vegetation characteristics, moisture condition, and index of continent to produce a population with similar genetic materials (within the distribution limit of the certain species of tree).

Mongolian forests are mountain forests
During the field survey, an inventory of seed stands and studies of vegetation and soil types were conducted, which enabled to produce detailed seed region maps. Digital Elevation Models with $30 \mathrm{~m}$ resolution and Satellite Images (Google Earth, Landsat) were used in the map processing.

with high altitudinal variation and dry growth conditions, hence, seed quality of the main tree species have been declining recently. For this reason, we did not divide seed regions into subregions. It is required to use seeds collected from local or neighboring regions/populations for the tree propagation and reforestation activities.

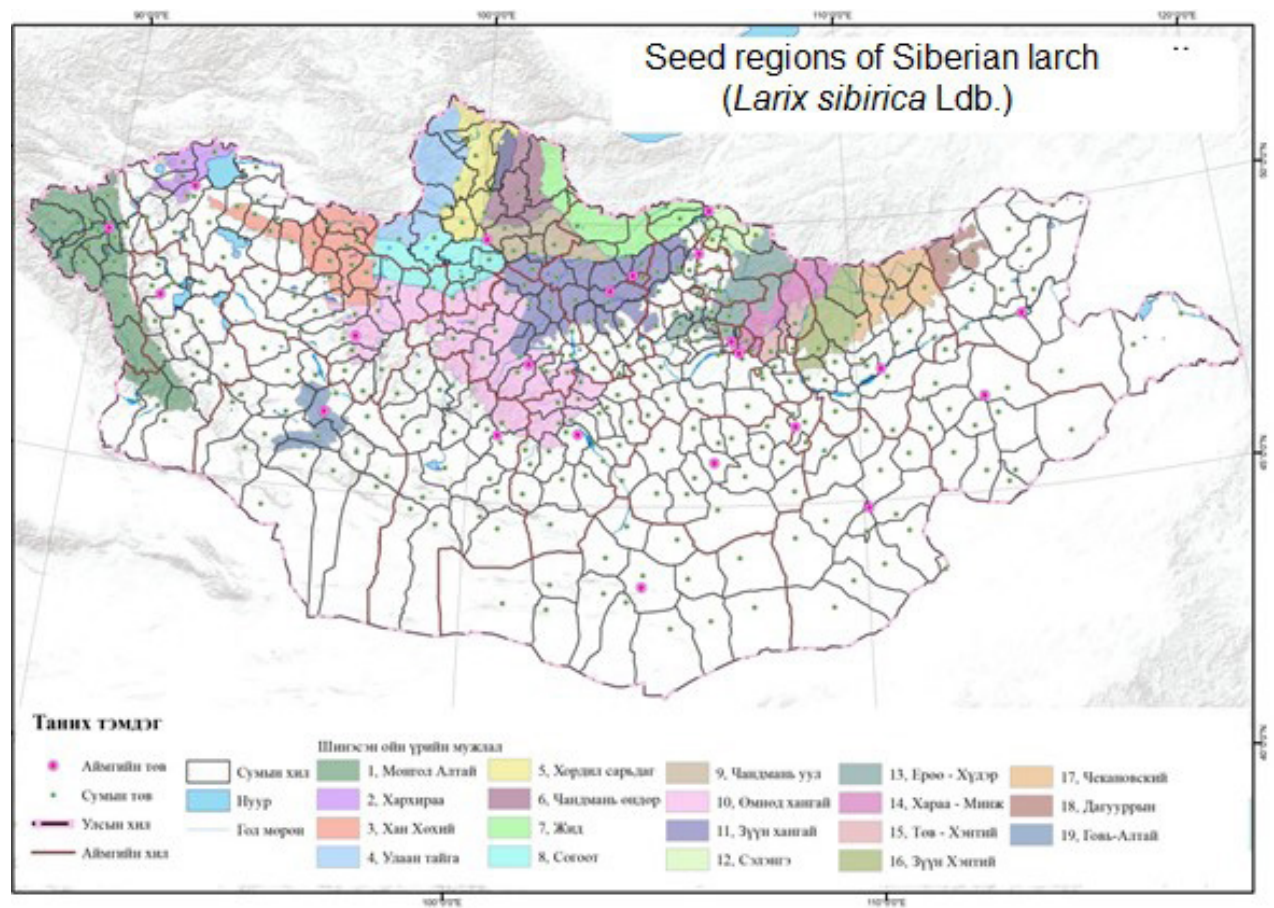

Figure 1. Siberian larch (Larix sibirica Ldb.) seed regions: 1. Mongol-Altai; 2. Kharkhiraa, 3. Khan Khokhii; 4. Ulaan taiga; 5. Khoridol Saridag; 6. Chandmani-Ondor; 7. Jid; 8. Sogoot; 9. Chandmani Uul; 10. Southern Khangai; 11. Eastern Khangai; 12. Selenge; 13. Yuroo-Khuder; 14. Kharaa-Minj; 15. Central Khentii; 16. Eastern Khentii; 17. Chekanowski; 18. Dahuur; 19. Gobi-Altai 
The research results identified 19 seed regions for Siberian larch (Larix sibirica Ldb.), Dahurian larch (Larix dahurica Turcz.), and Chekanowskii larch (Larix chekanowskii Szaf.); 12 regions for Scots pine (Pinus sylvestris L.); 9 regions for Siberian pine (Pinus sibirica Du Tour.); 6 regions for Siberian fir (Abies sibirica Ldb.), and; 9 seed regions for Siberian spruce (Picea obovata Ldb.) forests and a map with a scale of 1:1 000000 was developed which will serve as the base material for further seed management efforts with emphasis on conservation of forest genetic resources and tree breeding.

Seeds collected from the plain (steppe) forests can be used for almost all forest regions, however, seeds collected from mountainous regions can only be used within a 200-400 m range from the original altitude.
We identified 19 seed regions for Siberian larch and 12 regions for Scots pine, 9 regions for Siberian pine, 6 regions for Siberian fir and 9 seed regions for Siberian spruce forests. Each seed region is presented with information on possible regions to transfer seeds for transplanting, environment-climate conditions and regional forest characteristics, given in Tables 1-5.

Due to a lack of genetic-selection studies in Mongolia, these proposed forest seed regions can be considered a preliminary effort with the possibility to be updated and improved in the future, based on more detailed research results. These forest seed regions can be used as fundamental material for establishing a permanent forest seed supply, based on genetic-selection characteristics of the forests in different regions in Mongolia.

Table 1. Seed regions of Siberian larch forests

\begin{tabular}{|c|c|c|c|c|c|c|c|}
\hline № & Seed region & 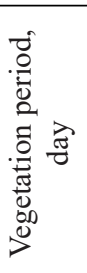 & 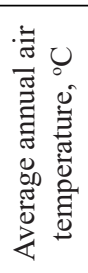 & 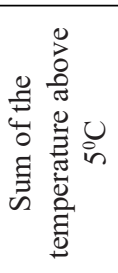 & 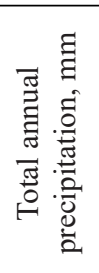 & 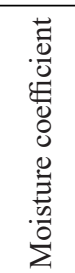 & $\begin{array}{c}\text { Average air } \\
\text { temperature, }{ }^{\circ} \mathrm{C} \\
{[\text { Conrad's index of }} \\
\text { continentally, \%] }\end{array}$ \\
\hline 1 & Mongol - Altai & 131 & -0.34 & 2147.1 & 107.0 & 0.65 & $\begin{array}{c}-21.4(17.3) . \\
{[89]}\end{array}$ \\
\hline 2 & Kharkhiraa & 137 & -1.75 & 2641.6 & 140.9 & 0.60 & $\begin{array}{c}-30.8(21.2) . \\
{[89]}\end{array}$ \\
\hline 3 & Khan Khokhii & 129 & -1.70 & 1956.9 & 191.4 & 1.32 & $\begin{array}{c}-28.5(17.6) . \\
{[79]}\end{array}$ \\
\hline 4 & Ulaan taiga & 123 & -5.37 & 1592.4 & 272.2 & 1.96 & $\begin{array}{c}-30.1(15.2) . \\
{[87]}\end{array}$ \\
\hline 5 & $\begin{array}{l}\text { Khoridol Sari- } \\
\text { dag }\end{array}$ & 121 & -4.17 & 1490.9 & 238.1 & 2.1 & $\begin{array}{c}-25.9(14.5) . \\
{[80]}\end{array}$ \\
\hline 6 & $\begin{array}{l}\text { Chandmani - } \\
\text { Ondor }\end{array}$ & 127 & -2.94 & 1719.5 & 261.1 & 1.96 & $\begin{array}{c}-23.5(15.2) . \\
{[82]}\end{array}$ \\
\hline 7 & Jid & 143 & 0.16 & 2119.9 & 309.6 & 1.46 & $\begin{array}{c}-23.3(19.4) . \\
{[79]}\end{array}$ \\
\hline 8 & Sogoot & 141 & -2.75 & 1934.8 & 245.0 & 1.57 & $\begin{array}{c}-26.3(16.7) . \\
{[80]}\end{array}$ \\
\hline 9 & Chandmani Uul & 143 & -0.54 & 2247.1 & 305.9 & 1.63 & $\begin{array}{c}-23.7(17.6) . \\
{[79]}\end{array}$ \\
\hline 10 & $\begin{array}{l}\text { Southern } \\
\text { Khangai }\end{array}$ & 152 & -1.62 & 1861.8 & 261.9 & 1.78 & $\begin{array}{c}-22.9(15.4) . \\
{[69]}\end{array}$ \\
\hline
\end{tabular}




\begin{tabular}{|l|l|c|c|c|c|c|c|}
\hline 11 & $\begin{array}{l}\text { Eastern Khan- } \\
\text { gai }\end{array}$ & 168 & 0.02 & 2124.9 & 314.3 & 1.68 & $\begin{array}{c}-21.1(18.5) . \\
{[74]}\end{array}$ \\
\hline 12 & Selenge & 127 & -1.33 & 1569.5 & 311.5 & 1.45 & $\begin{array}{c}-27.8(19.7) . \\
{[94]}\end{array}$ \\
\hline 13 & Yuroo - Khuder & 139 & -0.32 & 1962.8 & 297.7 & 1.42 & $\begin{array}{c}-24.6(19.4) . \\
{[85]}\end{array}$ \\
\hline 14 & Kharaa - Minj & 132 & -1.31 & 1929.0 & 312.6 & 1.94 & $\begin{array}{c}-22.8(16.8) . \\
{[76]}\end{array}$ \\
\hline 15 & Central Khentii & 129 & -1.19 & 1840.8 & 292.2 & 1.91 & $\begin{array}{c}-22.6(16.4) . \\
{[73]}\end{array}$ \\
\hline 16 & Eastern Khentii & 148 & -1.55 & 2149.9 & 332.9 & 1.81 & $\begin{array}{c}-20.1(17.9) . \\
{[76]}\end{array}$ \\
\hline 17 & Chekanovski & 156 & 0.11 & 2470.9 & 319.8 & 1.55 & $\begin{array}{c}-19.8(19.0) . \\
{[76]}\end{array}$ \\
\hline 18 & Daguur & 154 & 0.38 & 2429.7 & 334.9 & 1.59 & $\begin{array}{c}-19.6(19.5) . \\
{[74]}\end{array}$ \\
\hline 19 & Gobi-Altai & 168 & 1.62 & 2810.1 & 142.0 & 0.91 & $\begin{array}{c}-22.5(21.6) . \\
{[75]}\end{array}$ \\
\hline
\end{tabular}

*Source: Meteorological and Environmental Monitoring Agency

Table 2. Siberian larch (Larix sibirica Ldb.) seed region covers the following soums in certain aimag territory

\begin{tabular}{|c|c|c|}
\hline \multicolumn{2}{|r|}{ Seed region } & \multirow{2}{*}{ Seed region includes the following territory of soums } \\
\hline No & Name & \\
\hline 1 & Mongol - Altai & $\begin{array}{l}\text { Nogoonnuur, Ulaankhus, Tsengel, Sagsai, Altan Tsogts, Buyant, Altai, Tolbo, } \\
\text { Deluun soums Bayan-Olgii aimag; Bulgan, Uyench soums, Khovd aimag; }\end{array}$ \\
\hline 2 & Kharkhiraa & Davst, Sagil, Turgen, Taryalan soums, Uvs aimag; \\
\hline 3 & Khan Khokhii & $\begin{array}{l}\text { Malchin, Khyargas, Tsagaan Khairkhan, Ondor Khangai, Baruunturuun, } \\
\text { Zuunkhangai soums Uvs aimag; Tes, Bayantes, Asgat, Bayan Khairkhan, } \\
\text { Songino, Tudevtei, Nomrog, Tsetsen Uu soums, Zavkhan aimag; }\end{array}$ \\
\hline 4 & Ulaan taiga & $\begin{array}{l}\text { Tsagaannuur, Renchynlkhumbe, Ulaan Uul, Bayanzurkh, Tsagaan Uul, Tset- } \\
\text { serleg soums, Khovsgol aimag; }\end{array}$ \\
\hline 5 & Khoridol Saridag & $\begin{array}{l}\text { Renchynlkhumbe, Khankh, Khatgal, Arbulag, Alag Erdene soums, Khvsgol } \\
\text { aimag; }\end{array}$ \\
\hline 6 & Chamdmani - Ondor & $\begin{array}{l}\text { Khankh, Tsagaan Uur, Chandmani Ondor, Alag Erdene, Erdenebulgan, Tünel } \\
\text { soums, Khovsgol aimag; }\end{array}$ \\
\hline 7 & Jid & $\begin{array}{l}\text { Tsagaan Uur, Erdenebulgan soums, Khovsgol aimag; Teshig, Selenge soums, } \\
\text { Bulgan aimag; Tushig, Tsagaan Nuur soums, Selenge aimag }\end{array}$ \\
\hline 8 & Sogoot & $\begin{array}{l}\text { Tsetserleg, Tsagaan Uul, Burentogtokh, Tomorbulag, Shine Ider soums, } \\
\text { Khovsgol aimag; Ikh Uul, Telmen soums, Zavkhan aimag; }\end{array}$ \\
\hline 9 & Chandmani - Uul & $\begin{array}{l}\text { Tunel, Erdenebulgan, Tosontsengel, Ikh Uul, Taryalan soums, Khovsgol } \\
\text { aimag; } \\
\text { Khutag Ondor soums, Bulgan aimag; }\end{array}$ \\
\hline
\end{tabular}




\begin{tabular}{|l|l|l|}
\hline 10 & $\begin{array}{l}\text { Yaruu, Ider, Tosontsengel, Ikh Uul, Tsagaan Khairhan, soums, Zavkhan } \\
\text { aimag; } \\
\text { Jargalant, Galt soums, Khovsgol aimag; Tsakhir, Taryat, Jargalant, Khangai, } \\
\text { Chuluut, Ikhtamir, Erdenemandal, Tovshruulekh, Tsenkher, Bulgan, Tsetser- } \\
\text { leg, Khotont soums, Arkhangai aimag; Chuluut, Erdenetsogt soums, Bayank- } \\
\text { hongor aimag; Bat Olziit, Khujirt, Kharkhorin, Uyanga, Zuunbayan Ulaan } \\
\text { soums, Bayankhongor aimag; }\end{array}$ \\
\hline 11 & Eastern Khangai & $\begin{array}{l}\text { Rashaant soum, Khovsgol aimag; Tsetserleg, Erdenemandal, Khairkhan, } \\
\text { Battsengel, Olziit soums, Arkhagai aimag; Bayan Agt, Khutag Ondor, Bugat, } \\
\text { Saikhan, Mogod, Khishig Ondor, Burug Khangai, Orkhon, Khangal soums, } \\
\text { Bulgan aimag; Bayan Ondor, Jargalant soums, Orkhon aimag; Baruubüren, } \\
\text { Khushaat, Sant soums, Selenge aimag; }\end{array}$ \\
\hline 12 & Selenge & Shaamar, Altanbulag, Khuder, Yüroo soums, Selenge aimag; \\
\hline 13 & Yuroo - Khuder & $\begin{array}{l}\text { Yuroo, Khuder, Bugant, Bayangol, Mandal soums, Selenge aimag; } \\
\text { Khongor, Shariin Gol soums, Darkhan Uul aimag; Jargalant, Bornuur, Bayan- } \\
\text { chandmani, Batsumber soums, Tov aimag; }\end{array}$ \\
\hline 14 & Kharaa - Minj & $\begin{array}{l}\text { Ulaanbaatar, Erdene, Mongonmorti soums, Tov aimag; Batshireet soum, } \\
\text { Khentii aimag; }\end{array}$ \\
\hline 15 & Central Khentii & Erdene, Mongonmorti, Bayandelger soums, Tov aimag; \\
\hline 16 & Eastern Khentii & Tsenkhermandal, Omnodelger, Batshireet soums, Khentii aimag; \\
\hline 17 & Checanovski & $\begin{array}{l}\text { Omnodelger, Binder, Bayan Adarga, Norovlin, Dadal soums, Khentii aimag; } \\
\text { Bayan Uul, Tsagaan Ovoo soums, Dornod aimag; }\end{array}$ \\
\hline 18 & Daguur & Bayan Uul, Bayandun soums, Dornod aimag; \\
\hline 19 & Gobi - Altai & Jargalan, Khaliun, Togrog soums, Gobi - Altai aimag; \\
\hline
\end{tabular}

Table 3. Seed regions of Scotch pine forests

\begin{tabular}{|c|c|c|c|c|c|c|c|}
\hline № & Seed region & 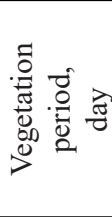 & 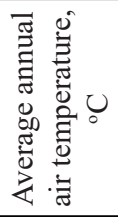 & 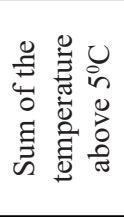 & 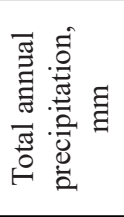 & 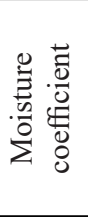 & $\begin{array}{c}\text { Average air } \\
\text { temperature, }{ }^{\circ} \mathrm{C} \\
{[\text { Conrad's index of }} \\
\text { continentally, } \% \text { ] }\end{array}$ \\
\hline 1 & Tsagaan - Uur & 132 & -3.9 & 1848.6 & 250.7 & 2.54 & $\begin{array}{c}-30.1(16.4) \\
{[89]}\end{array}$ \\
\hline 2 & Jid & 143 & 0.16 & 2119.9 & 309.6 & 1.46 & $\begin{array}{c}-23.3(19.4) \\
{[79]} \\
\end{array}$ \\
\hline 3 & Burengiin Nuruu & 149 & -0.01 & 1997.1 & 318.0 & 1.61 & $\begin{array}{c}-22.5(19.3) \\
{[79]}\end{array}$ \\
\hline 4 & Bayan - Khan uul & 151 & 0.52 & 1718.0 & 275.8 & 1.23 & $\begin{array}{c}-23.2(20.6) \\
{[85]}\end{array}$ \\
\hline 5 & Yuroo - Khuder & 133 & -1.01 & 1565.0 & 334.3 & 1.55 & $\begin{array}{c}-26.8(19.4) . \\
{[91]}\end{array}$ \\
\hline 6 & Kharaa Minj & 146 & -0.34 & 2050.5 & 292.2 & 1.45 & $\begin{array}{c}-23.2(19.1) . \\
{[81]}\end{array}$ \\
\hline 7 & Shariin Gol & 139 & -0.58 & 1902.5 & 298.7 & 1.39 & $\begin{array}{c}-26.2(19.8) . \\
{[89]}\end{array}$ \\
\hline 8 & Jargalant & 163 & 0.11 & 2301.2 & 299.0 & 1.49 & $\begin{array}{c}-21.6(18.6) \\
{[75]}\end{array}$ \\
\hline
\end{tabular}




\begin{tabular}{|c|l|c|c|c|c|c|c|}
\hline 9 & Bayanchandmani & 159 & 0.01 & 2184.6 & 277.1 & 1.47 & $\begin{array}{c}-20.0(17.7) . \\
{[71]}\end{array}$ \\
\hline 10 & Eastern Khentii & 162 & 0.03 & 2422.1 & 286.9 & 1.35 & $\begin{array}{c}-21.5(19.5) \\
{[82]}\end{array}$ \\
\hline 11 & Ereenii Nuruu & 178 & -0.21 & 2363.8 & 322.8 & 1.49 & $\begin{array}{c}-20.6(18.5) . \\
{[77]}\end{array}$ \\
\hline 12 & Khalkh Gol & 183 & 0.01 & 2672.7 & 350.2 & 1.46 & $\begin{array}{c}-28.9(20.9) . \\
{[98]}\end{array}$ \\
\hline
\end{tabular}

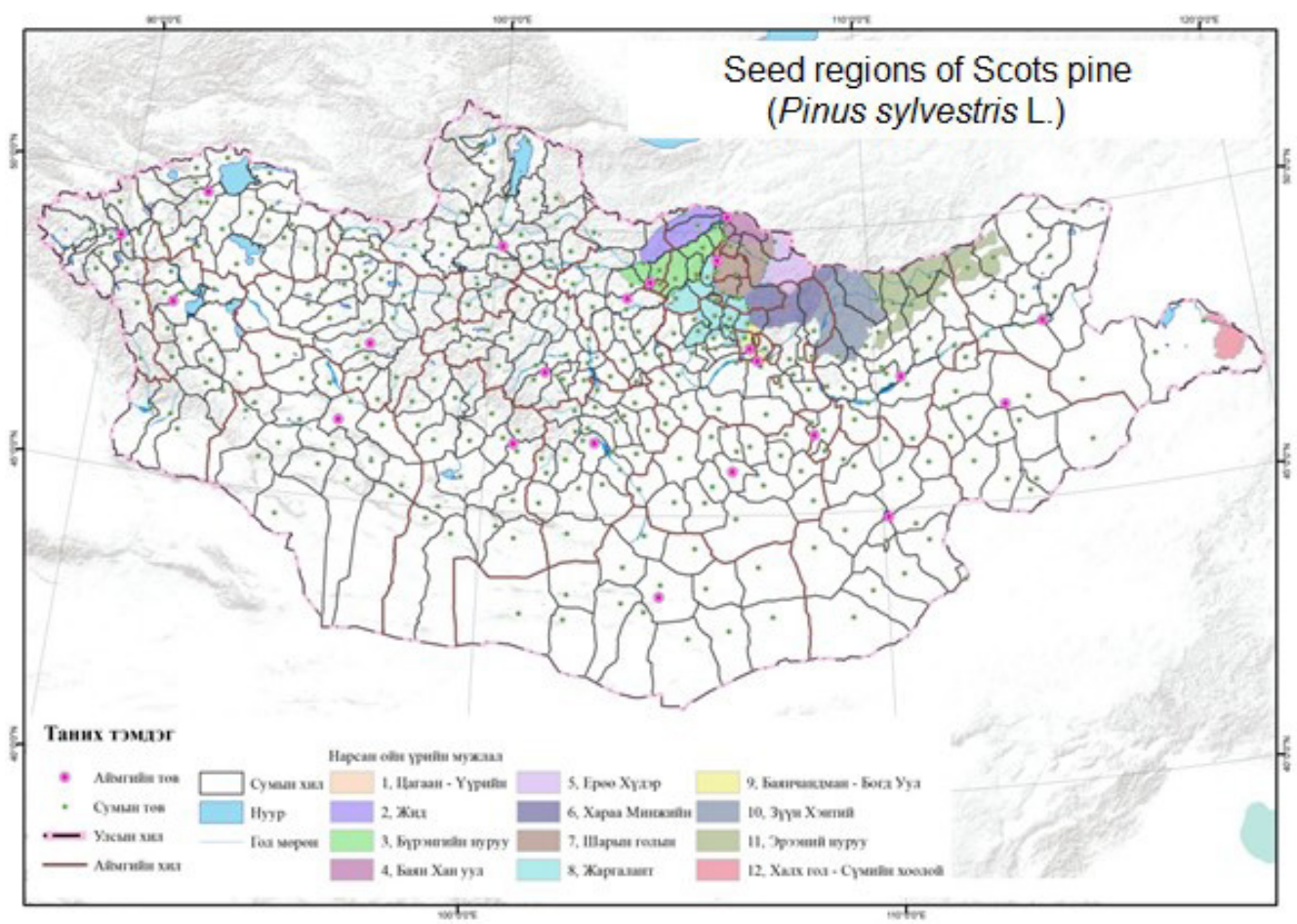

Figure 2. Scots pine (Pinus sylvestris L) seed regions: Tsagaan - Uur; 2. Jid; 3. Burengiin Nuruu; 4. Bayan - Khan Uul; 5. Ybroo - Khuder; 6. Kharaa-Minj; 7. Shariin Gol; 8. Jargalant; 9. Bayanchandmani; 10. Eastern Khentii; 11. Ereenii Nuruu; 12. Khalkh Gol

Table 4. Scots pine (Pinus sylvestris L.) seed region covers the following soums in certain aimag territory

\begin{tabular}{|l|l|l|}
\hline \multicolumn{2}{|c|}{ Seed region name } & \multicolumn{1}{c|}{ Seed region includes the following territory of soums } \\
\hline No & \multicolumn{1}{|c|}{ Name } & \multicolumn{1}{|c|}{ Tsagaan - Uur soum, Khovsgol aimag } \\
\hline 1 & Tsagaan - Uur & $\begin{array}{l}\text { Khutag-Ondor, Selenge, Khilgant soums, Bulgan aimag; Tushig, } \\
\text { Tsagaannuur soums, Selenge aimag. }\end{array}$ \\
\hline 2 & Jid & $\begin{array}{l}\text { Bugat, Saikhan, Khilgant soums, Bulgan aimag; Bayan Ondor, Jar- } \\
\text { galant soum, Orkhon aimag; Baruunburen, Sant, Khushaat, Zuunburen } \\
\text { soums, Selenge aimag. }\end{array}$ \\
\hline 4 & Burengiin Nuruu & Shaamar, Altanbulag soums, Selenge aimag. \\
\hline
\end{tabular}




\begin{tabular}{|l|l|l|}
\hline 5 & Yuroo - Khuder & Yuroo, Khuder, Bugant soums, Selenge aimag. \\
\hline 6 & Kharaa - Minj & $\begin{array}{l}\text { Mandal soum, Selenge aimag; Batsumber soum, Tov aimag; Batshireet } \\
\text { soum, Khentii aimag; }\end{array}$ \\
\hline 7 & Shariin Gol & $\begin{array}{l}\text { Jargalant, Yeruu, Bugant, Bayangol soums, Selenge aimag; Khongor, } \\
\text { Shariin Gol soums, Darkhan aimag; }\end{array}$ \\
\hline 9 & Jargalant & Jargalant, Bornuur, Chandmani, Batsumber soums, Tov aimag; \\
\hline 10 & Bayan- Adarga & Bogdkhan uul, Ulaanbaatar \\
\hline 11 & Yuroonii nuruu & $\begin{array}{l}\text { Binder, Bayan Adarga, Norovlin, Dadal soums, Khentii aimag; Bayan } \\
\text { Uul, Bayandun soums,Dornod aimag; }\end{array}$ \\
\hline 12 & Sumiin Khooloi & Khalkh Gol soum, Dornod aimag; \\
\hline
\end{tabular}

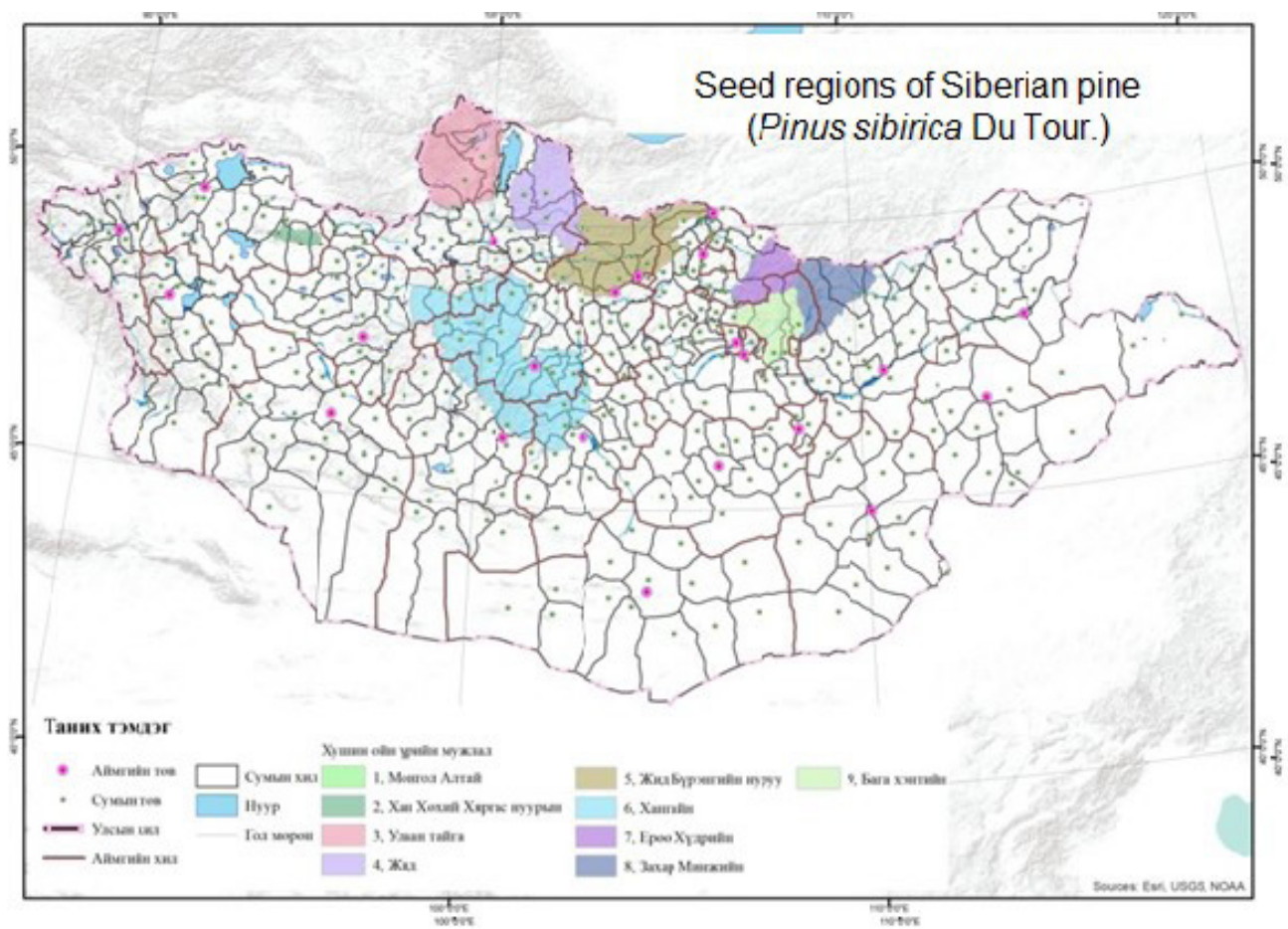

Figure 3. Siberian pine (Pinus sibirica Du Tour.) seed regions: 1. Mongol-Altai; 2. Khan KhokhiiKhyargas lake; 3. Ulaan taiga; 4. Jid; 5. Jid Burengiin Nuruu; 6. Khangain Nuruu; 7. Yuroo-Khuder; 8. Zakhar-Minj; 9. Baga Khentii 
Table 5. Seed regions of Siberian pine

\begin{tabular}{|c|c|c|c|c|c|c|c|}
\hline № & Seed region & 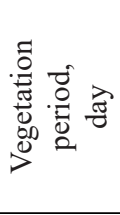 & 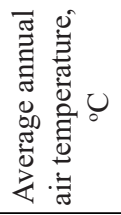 & 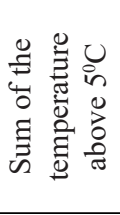 & 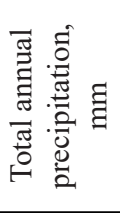 & 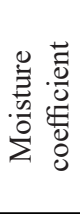 & $\begin{array}{c}\text { Average air } \\
\text { temperature, }{ }^{\circ} \mathrm{C} \\
{[\text { Conrad's index of }} \\
\text { continentally, \%] }\end{array}$ \\
\hline 1 & Mongol - Altai & 131 & -0.34 & 2147.1 & 107.0 & 0.65 & $\begin{array}{c}-21.4(17.3) . \\
{[89]}\end{array}$ \\
\hline 2 & $\begin{array}{l}\text { Khan Khokhii - } \\
\text { Khyargas lake }\end{array}$ & 137 & -1.75 & 2641.6 & 140.9 & 0.60 & $\begin{array}{c}-30.8(21.2) . \\
{[89]}\end{array}$ \\
\hline 3 & Ulaan taiga & 123 & -5.37 & 1592.4 & 272.2 & 1.96 & $\begin{array}{c}-30.1(15.2) . \\
{[87]}\end{array}$ \\
\hline 4 & Jid & 143 & 0.16 & 2119.9 & 309.6 & 1.46 & $\begin{array}{c}-23.3(19.4) . \\
{[79]}\end{array}$ \\
\hline 5 & $\begin{array}{l}\text { Jid Burengiin } \\
\text { Nuruu }\end{array}$ & 149 & -0.01 & 1997.1 & 318.0 & 1.61 & $\begin{array}{c}-22.5(19.3) . \\
{[79]}\end{array}$ \\
\hline 6 & Khangai Nuruu & 139 & -0.32 & 1962.8 & 297.7 & 1.42 & $\begin{array}{c}-24.6(19.4) . \\
{[85]}\end{array}$ \\
\hline 7 & Yuroo - Khuder & 133 & -1.01 & 1565.0 & 334.3 & 1.55 & $\begin{array}{c}-26.8(19.4) . \\
{[91]}\end{array}$ \\
\hline 8 & Zakhar Minj & 146 & -0.34 & 2050.5 & 292.2 & 1.45 & $\begin{array}{c}-23.2(19.1) . \\
{[81]}\end{array}$ \\
\hline 9 & Baga Khentii & 132 & -1.31 & 1929.0 & 312.6 & 1.94 & $\begin{array}{c}-22.8(16.8) . \\
{[76]}\end{array}$ \\
\hline
\end{tabular}

*Source: Meteorological and Environmental Monitoring Agency

Table 6. Siberian pine (Pinus sibirica Du Tour.) seed region covers the following soums in certain aimag territory

\begin{tabular}{|l|l|l|}
\hline \multicolumn{2}{|c|}{ Seed region name } & \multicolumn{1}{|c|}{ Seed region includes the following territory of soums } \\
\hline No & \multicolumn{1}{|c|}{ Name } & \multicolumn{1}{|c|}{ Tsengel soum, Bayan-Olgii aimag; } \\
\hline 1 & Mongol-Altai & $\begin{array}{l}\text { Tsagaan Khairkhan, Ondorkhangai, Zuunkhangai soums, Uvs aimag; } \\
\text { Tes soum, Zavkhan aimag; }\end{array}$ \\
\hline 3 & Khyan Khokhii & $\begin{array}{l}\text { Tsagaannuur, Renchinlkhumbe, Ulaan Uul, Bayanzurkh, Tsetserleg } \\
\text { soums, Khovsgol aimag; }\end{array}$ \\
\hline 4 & Jid & $\begin{array}{l}\text { Tsagaanuur, Chandmani Ondor, Erdenebulgan, Taryalan soums } \\
\text { Khovsgol aimag; Teshig soum, Bulgan aimag; }\end{array}$ \\
\hline 5 & Jid - Burengiin Nuruu & $\begin{array}{l}\text { Bayan Agt, Saikhan, Khutag Ondor, Selenge, Teshig soums, Bulgan } \\
\text { aimag; Baruunburen, Tushig, Tsagaannuur soums, Selenge aimag; }\end{array}$ \\
\hline 6 & Khangai & $\begin{array}{l}\text { Tosontsengel soum, Zavkhan aimag; Jargalant, Galt soums Khovs- } \\
\text { gol aimag; Tsakhir, Taryat, Khangai, Chuluut, Ikh Tamir, Jargalant, } \\
\text { Bulgan, Tsenkher, Tovshruulekh soums, Arkhangai aimag; Bat Olziit, } \\
\text { Khujirt, Uyanga soums, Ovorkhangai aimag; }\end{array}$ \\
\hline 7 & Yuroo - Khuder & Yuroo, Khuder soums, Selenge aimag; \\
\hline 8 & Zakhar - Minj & $\begin{array}{l}\text { Mongonmorti soum, Töv aimag; } \\
\text { Tsenkhermandal, Batshireet, Binder soums, Khentii aimag; }\end{array}$ \\
\hline 9 & Baga Khentii & Ulaanbaatar, Erdene, Bayandelger soums, Tov aimag; \\
\hline
\end{tabular}




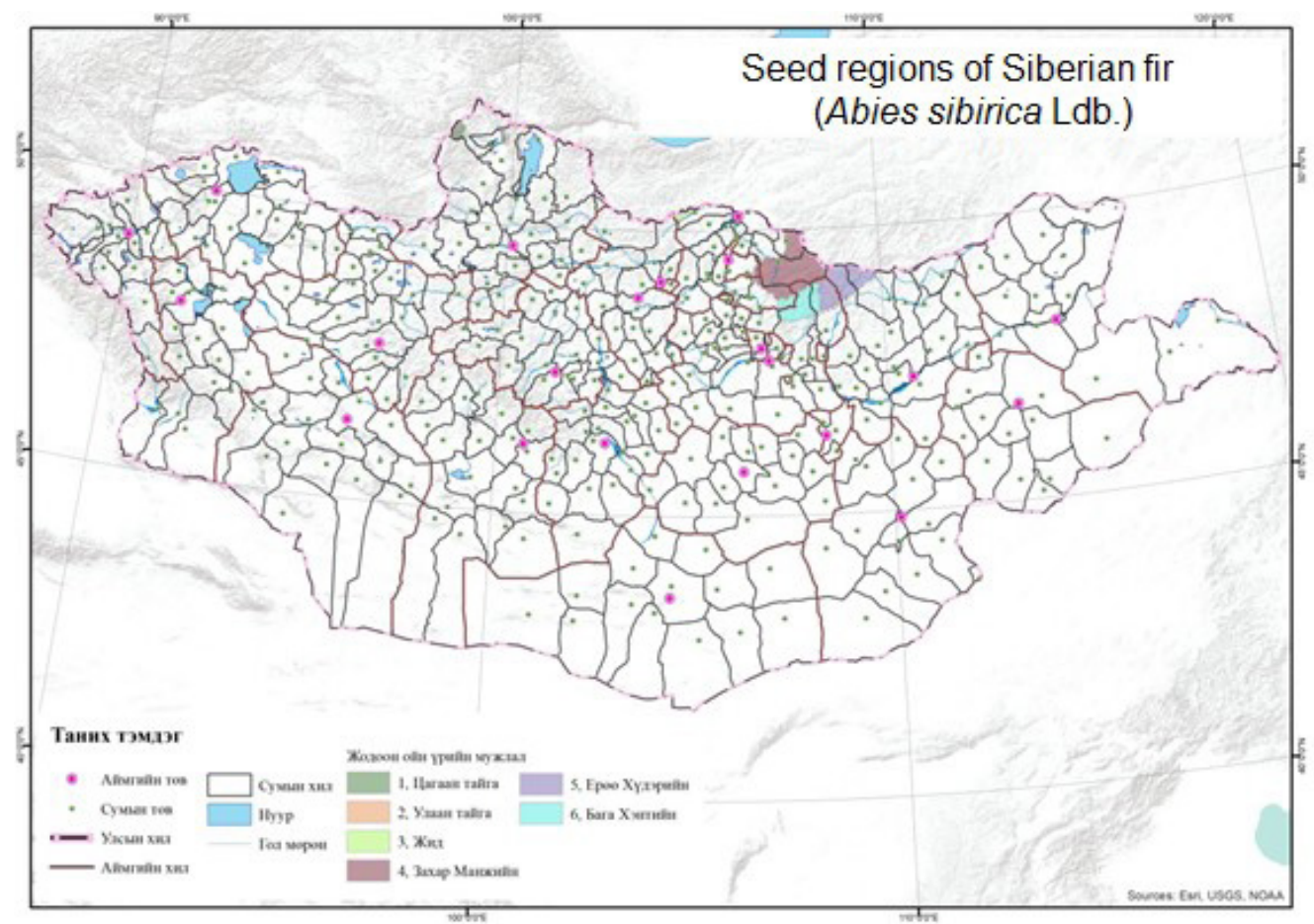

Figure 4. Siberian fir (Abies sibirica Ldb.) seed regions: 1. Tsagaan Taiga; 2. Ulaan Taiga; 3. Jid; 4. Zakhar Minj; 5. Ybroo - Khuder; 6. Baga Khentii

Table 7. Seed regions of Siberian fir forests

\begin{tabular}{|c|c|c|c|c|c|c|c|}
\hline № & Seed region & 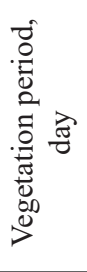 & 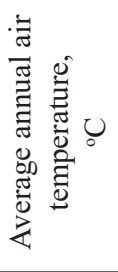 & 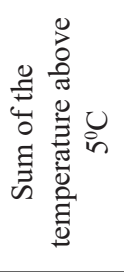 & 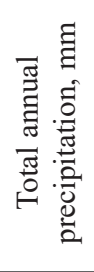 & 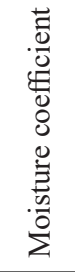 & $\begin{array}{c}\text { Average air } \\
\text { temperature, }{ }^{\circ} \mathrm{C} \\
{[\text { Conrad's index of }} \\
\text { continentally, \%] }\end{array}$ \\
\hline 1 & Tsagaan taiga & 121 & -6.67 & 1536.3 & 218.3 & 1.58 & $\begin{array}{c}-34.4(15.7) . \\
{[97]}\end{array}$ \\
\hline 2 & Ulaan taiga & 123 & -5.37 & 1592.4 & 272.2 & 1.96 & $\begin{array}{c}-30.1(15.2) \\
{[87]} \\
\end{array}$ \\
\hline 3 & Jid & 143 & 0.16 & 2119.9 & 309.6 & 1.46 & $\begin{array}{c}-23.3(19.4) . \\
{[79]}\end{array}$ \\
\hline 4 & Zakhar Minj & 133 & -1.01 & 1565.0 & 334.3 & 1.55 & $\begin{array}{c}-26.8(19.4) . \\
{[91]} \\
\end{array}$ \\
\hline 5 & $\begin{array}{l}\text { Yüroo - } \\
\text { Khuder }\end{array}$ & 146 & -0.34 & 2050.5 & 292.2 & 1.45 & $\begin{array}{c}-23.2(19.1) . \\
{[81]}\end{array}$ \\
\hline 6 & Baga Khentii & 132 & -1.31 & 1929.0 & 312.6 & 1.94 & $\begin{array}{c}-22.8(16.8) \\
{[76]} \\
\end{array}$ \\
\hline
\end{tabular}


Table 8. Siberian fir (Abies sibirica Ldb.). seed region covers the following soums in certain aimag territory

\begin{tabular}{|c|c|c|}
\hline \multicolumn{2}{|r|}{ Seed region } & \multirow{2}{*}{ Seed region includes the following territory of soums } \\
\hline № & Name & \\
\hline 1 & Tsagaan taiga & Tsagaannuur soum, Khovsgol aimag; \\
\hline 2 & Ulaan taiga & Renchinlkhümbe soum, Khovsgol aimag; \\
\hline 3 & Jid & Tushig, Tsagaannuur soum, Selenge aimag; \\
\hline 4 & Yuroo - Khuder & Yuroo, Khuder, Bugant, Mandal soums, Selenge aimag; \\
\hline 5 & Zakhar - Minj & $\begin{array}{l}\text { Mandal soum, Selenge aimag; Mongnmorti soum, Tov aimag; } \\
\text { Batshireet soum, Khentii aimag; }\end{array}$ \\
\hline 6 & Baga Khentii & Mongonmorti soum, Tov aimag; \\
\hline
\end{tabular}

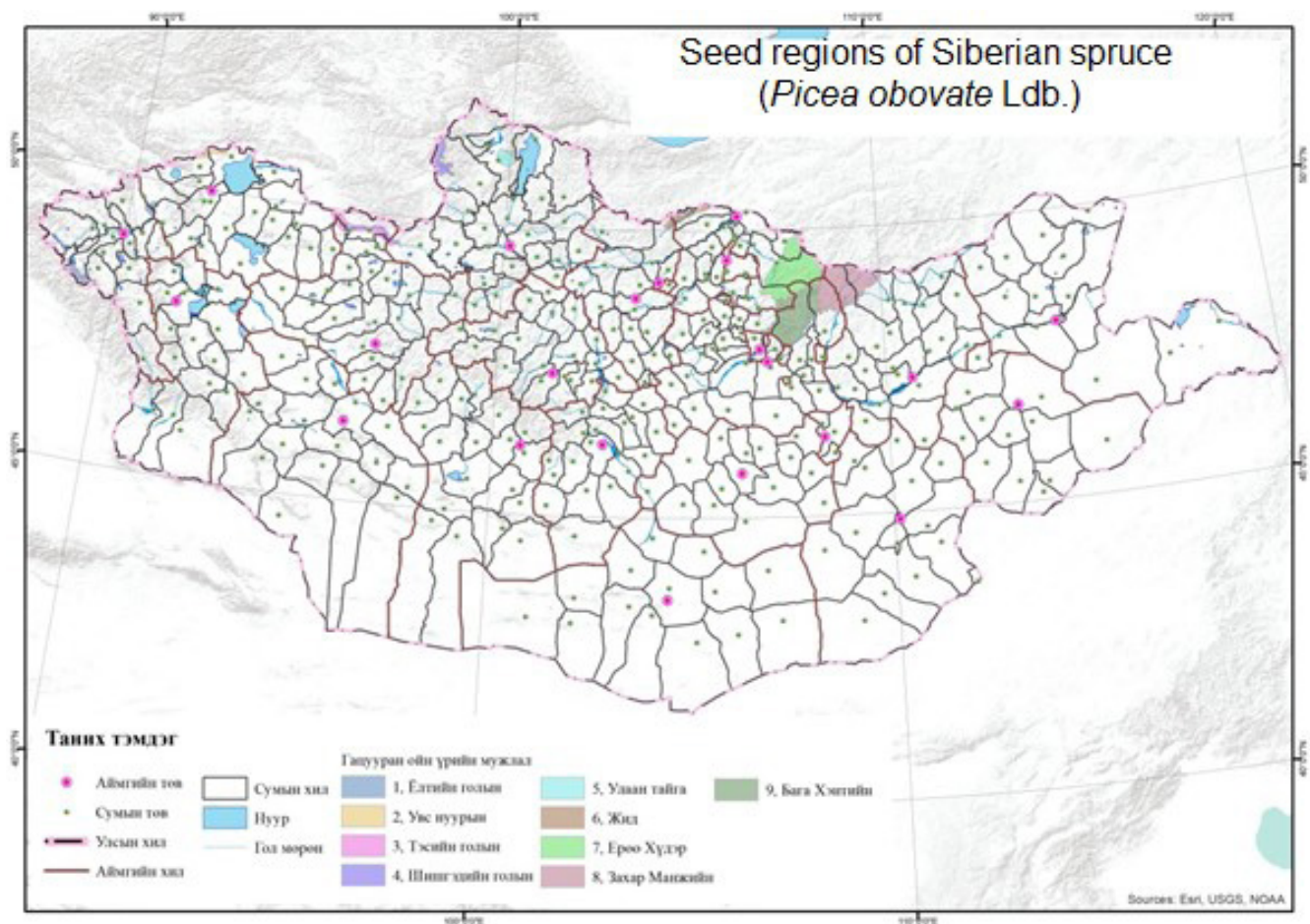

Figure 5. Siberian spruce (Picea obovate Ldb.) seed regions: 1. Yoltiin Gol; 2. Uvs Nuur; 3. Tesiin Gol; 4. Shishgediin Gol; 5. Ulaan Taiga; 6. Jid; 7. Yuroo - Khuder 8. Zakhar-Minj; 9. Baga Khentii 
Table 9. Seed regions of Siberian spruce forests

\begin{tabular}{|c|c|c|c|c|c|c|c|}
\hline № & Seed region & 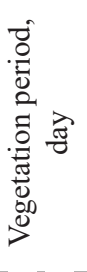 & 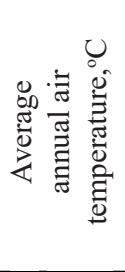 & 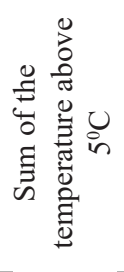 & 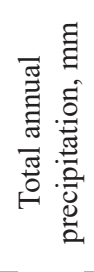 & 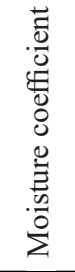 & $\begin{array}{c}\text { Average air } \\
\text { temperature, }{ }^{\circ} \mathrm{C} \\
{[\text { Conrad's index of }} \\
\text { continentally, } \%]\end{array}$ \\
\hline 1 & Yoltiin Gol & 131 & -0.34 & 2147.1 & 107.0 & 0.65 & $\begin{array}{c}-21.4(17.3) . \\
{[89]}\end{array}$ \\
\hline 2 & Uvs Nuur & 155 & -1.41 & 2848.2 & 109.2 & 0.42 & $\begin{array}{c}-31.6(22.4) \\
{[93]}\end{array}$ \\
\hline 3 & Tesiin Gol & 130 & -0.39 & 2002.2 & 185.9 & 1.08 & $\begin{array}{c}-32.4(17.8) \\
{[88]}\end{array}$ \\
\hline 4 & Shishgediin Gol & 121 & -6.67 & 1536.3 & 218.3 & 1.58 & $\begin{array}{c}-34.4(15.7) \\
{[97]}\end{array}$ \\
\hline 5 & Ulaan Taiga & 123 & -5.37 & 1592.4 & 272.2 & 1.96 & $\begin{array}{c}-30.1(15.2) \\
{[87]}\end{array}$ \\
\hline 6 & Jid & 143 & 0.16 & 2119.9 & 309.6 & 1.46 & $\begin{array}{c}-23.3(19.4) \\
{[79]}\end{array}$ \\
\hline 7 & Yuroo - Khuder & 133 & -1.01 & 1565.0 & 334.3 & 1.55 & $\begin{array}{c}-26.8(19.4) . \\
{[91]}\end{array}$ \\
\hline 8 & Zakhar Minj & 146 & -0.34 & 2050.5 & 292.2 & 1.45 & $\begin{array}{c}-23.2(19.1) \\
{[81]}\end{array}$ \\
\hline 9 & Baga Khentii & 132 & -1.31 & 1929.0 & 312.6 & 1.94 & $\begin{array}{c}-22.8(16.8) \\
{[76]}\end{array}$ \\
\hline
\end{tabular}

*Source: Meteorological and Environmental Monitoring Agency

Table 10. Siberian spruce (Picea obovata Ldb.) seed region covers the following soums in certain aimag territory

\begin{tabular}{|l|l|l|}
\hline \multicolumn{2}{|l|}{ Seed region } & \multirow{2}{*}{ Seed region includes the following territory of soums } \\
\cline { 1 - 2 } № & Name & Altai soum, Bayan-Olgii aimag; \\
\hline 1 & Yoltiin Gol & Davst soum, Uvs aimag; \\
\hline 3 & Tesiin Gol & Tes, Bayantes soums, Zavkhan aimag; \\
\hline 4 & Shishigtiin Gol & Tsagaannuur soum, Khovsgol aimag; \\
\hline 5 & Ulaan Taiga & Renchinlkhümbe soum, Khovsgol aimag; \\
\hline 6 & Jid & Tushig, Tsagaannuur soums, Selenge aimag; \\
\hline 7 & Yuroo - Khuder & Yuroo, Khuder, Bugant soums Selenge aimag; \\
\hline 8 & Zakhar - Minj & $\begin{array}{l}\text { Mongonmorti soums, Tov aimag; Tsenkhermandal, Batshireet, Binder } \\
\text { Delgerkhaan soums, Khentii aimag; }\end{array}$ \\
\hline 9 & Baga Khentii & $\begin{array}{l}\text { Capital (metropolis) Ulaanbaatar; Erdene, Bayandelger soums, Tov } \\
\text { aimag; }\end{array}$ \\
\hline
\end{tabular}




\section{CONCLUSIONS}

We have identified 19 seed regions for Siberian larch (Larix sibirica Ldb.), Dahurian larch (Larix dahurica Turcz.), Chekanowskii larch (Larix Chekanowskii Szaf.); 12 regions for Scots pine (Pinus sylvestris L.); 9 regions for Siberian pine (Pinus sibirica Du Tour.); 6 regions for Siberian fir (Abies sibirica Ldb.); and 9 seed regions for Siberian spruce forests (Picea obovata Ldb.), and have developed a map on a scale of 1:1000 000 which will serve as a basic material for further seed management efforts with an emphasis on conservation of forest genetic resources and tree breeding in coniferous forests in Mongolia.

Acknowledgements. This research has been conducted under the "Conservation of forest genetic resources" project and we would like to express our sincere appreciation to the Department of Forest Policy and Coordination, Ministry of Environment and Tourism, Forest Research, Development Center, Institute of Geography and Geoecology, Mongolian Academy of Sciences and Monconsult Co., Ltd. We also thank Misha Goforth for language improvement of this article.

\section{REFERENCES}

1. Barchenkov A. P., Milyutin L. I., Jamiyansüren S., Variability of Siberian larch (Larix sibirica Ledeb.) generative organs in mountain populations in Mongolia and adjacent regions of Russia, Siberian Journal of Forest Science. N. 4: 2015, pp. 58-64, (In Russian)

2. DeFries, S. M. G. et al. Pan-European strategy for genetic conservation units. Rome: European Forest Genetic Resources Programme (EUFORGEN), Biodivesity International. 2015, p. 42.

3. Dorjgotov D. Soil of Mongolia, Thesis, M.,1992, p. 51, (In Russian)

4. Dugarjav, Ch. Larch forests in Mongolia, Ulaanbaatar, 2006, p. 318, (In Mongolian)

5. Forests of the Mongolian People's Republic (Larch forest of Central Khangai). Nauka, Novosibirsk, 1983, p. 149. (in Russian)

6. Forests of the Mongolian People's Republic (Larch forest of Eastern Khentii). Nauka, Moskow, 1988, p. 177. (in Russian)

7. Forests of the Mongolian People's Republic (Geography and topology). Nauka, Moskow, 1978, p. 128. (in Russian)

8. Hamann, A., Gylander, T. and Chen P. Y. Developing seed zones and transfer guidelines with multivariate regression trees. Berlin, Heidelberg: Springer. Tree Genetics \& Genomes 7: 2010, pp. 399-408.

9. Jamyansüren, S., Milyutin L. I. Variability of seed quality in the Mongolian populations Larix sibirica and Pinus sylvestris. Conservation of forest genetic resources in Siberia. Proceedings of $3^{\text {rd }}$ international conference. Krasnoyarsk, pp. 45-46, (In Russian)

10. Jamyansüren, S. Difference of Larix gemlinii Rupr, Larix sibirica Ldb- Eastern Khentii. Journal of Agriculture. UB., 1985, pp. 22-24

11. Jamyansüren, S. Some issues on seed management of Siberian larch. Proceeding of the conference of forest restoration and reforestation in Republic of Mongolia. Ulaanbaatar, pp. 16-19. (In Mongolian)

12. Milyutin L. I., Jamyansüren, S. Barchenkov A. P. Variability of Siberian larch (Larix sibirica Ledeb.) Khangai in Mongolia / Lesovedenie (Russian journal of forest science). 2: 2015, pp.24-27, (In Russian)

13. Milyutin L. I., Suntsov A.V., Jamyansüren, S. Genetics selection features of the main 
forest species of the Eastern Khentei. Nauka, Moskow, 1988, pp. 75-118. (in Russian)

14. Natsagdorj, L. Assessment of forest seed quality and weather conditions. Siberian larch and pine seed quality analysis conducted by Forest Seed Laboratory.UB. 2012, pp. 79 97, (In Mongolian)

15. Parker, W. H. and van Niejenhuis, A. Regression-based focal point seed zones for Picea mariana from northwestern Ontario. Canadian Journal of Botany 74 (8): 1996, pp. 1227 1235.

16. Paul, M. et al. Concept for the Conservation and Sustainable Utlization of Forest Genetic Resources in the Federal Republic of Germany. Bonn: Federal Ministry of Food, Agriculture and Consumer Protection. 2010, p. 76.

17. Udval, B. Growth characteristics, seed crop and seed quality of seed stands of Scotch pine (Pinus sylvestris L.). Dissertation. Ulaanbaatar. 2014, p. 98. (In Mongolian) 